Results Development of a free online course hosted by University of Northampton. Participants:

- $97 \%$ agreed/strongly agreed that the module had been organised in a way that helped them to learn

- $68 \%$ felt that they had significantly increased their knowledge of MND and a further 30\% believed they had increased their knowledge

- Following the course 94\% felt more confident about caring for someone living with MND.

Reasons for completing the course varied: $47 \%$ indicated they were caring for someone with MND, and 32\% were undertaking it purely for professional development. A significant proportion of completions were outside core hours: $31 \%$ 7 pm - midnight and 11\% midnight - 7 am; a requirement not easily accommodated by training. The course has been used to provide wholesale staff training.

Conclusion This module has approximately 700 completions to date. It provides a comprehensive, practical introduction to MND and has been well received by participants. It could usefully be employed within hospices to support the delivery of outstanding care.

\section{P-255 THE IMPLEMENTATION OF PROJECT ECHO INTO NORTH EAST ESSEX}

Caroline Vince, Imelda Hodgkinson, Christine Aylott, Karen Chumbley, Jacquie Pamphilon, Jennifer Chandler. St Helena Hospice, Colchester, UK

10.1136/bmjspcare-2018-hospiceabs.280

Background Project ECHO ${ }^{\circledR}$ (Extension of Community Healthcare Outcomes) is a virtual environment learning tool, used within a community of practice among care providers, to facilitate a more comprehensive seamless experience for care staff, patients and families.

Aim The concept of ECHO in this instance is being utilised to disseminate knowledge and best practice within the palliative care community to enable cohesive working. This united approach lends itself to problem solving and improving the quality of decision making within the healthcare sector with emphasis on collaboration and shared focus, to enhance standards of care, in a dynamic and revolutionary manner.

Method Utilising the Gold Standards Framework workshops for nursing and residential homes, we introduced the concept of ECHO by demonstrating a video example plus an in-house demonstration. As a consequence, we gained buy-in and will run a pilot dividing the nursing and residential homes into two groups of eight and delivering six ECHO sessions over a period of six months from July 2018.

Results These sessions will be based on the identified learning needs of the 16 nursing and residential homes. The first three sessions will be based on communication, symptom management at the end of life and advance care planning. A guest speaker will provide a 20 min presentation on the chosen subject which will set the tone for the remainder of the discussion. Two of the nursing and residential homes will provide case studies relating to the theme of the session with active participation from the expert panel at the hospice hub and all participants.

Conclusion From initial observation, the idea of ECHO has captured the enthusiasm and willingness of shared learning to improve patient experience with 16 out of the 24 nursing and residential homes voicing an interest in engaging with the opportunity to participate in ECHO.

\section{P-256 DESIGNING AN E-ELCA LEARNING PATH FOR SPECIALISTS IN PALLIATIVE CARE}

${ }^{1,2,3}$ Richard Kitchen, ${ }^{4}$ Emily Curran. 'University Hospitals of Coventry and Warwickshire, Coventry, UK; ${ }^{2}$ The Myton Hospices, Warwick, UK; ${ }^{3}$ The Association for Palliative Medicine, Fareham, UK; ${ }^{4}$ Health Education Yorkshire and the Humber, Leeds, UK

\subsection{6/bmjspcare-2018-hospiceabs.281}

Background e-ELCA (end of life care for all) is an e-learning programme from e-Learning for Health, delivering palliative and end of life care education. It was originally developed to support the Department of Health's End of Life Care Strategy, being designed to deliver education to the wider NHS workforce. More recently, there has also been a focus on offering educational opportunities to specialists in palliative care. Of note, e-ELCA is comprised of over 160 sessions and utilises 'learning paths' to allow users to identify sessions that will be helpful for their learning.

Aim To design an e-ELCA learning path for specialists in palliative care. This would allow this group to easily identify e-ELCA sessions that are relevant for their own learning.

Methods The JRCPTB (Joint Royal College of Physicians Training Board, 2014) speciality training curriculum for palliative medicine is used in the training of palliative medicine specialty registrars. The curriculum contains many sections including physical care, communication and ethics. Educational resources that support this curriculum will be helpful for registrars, but are also likely to be relevant for other specialists in palliative care. Therefore, e-ELCA sessions were mapped to the JRCPTB speciality training curriculum for palliative medicine (2010 with amendments 2014)1. This process was carried out by a specialty registrar in palliative medicine, with this work then reviewed by the e-ELCA clinical lead.

Results Initially 34 e-ELCA sessions were identified that mapped to the curriculum. Following further review, 10 of these were deemed to too basic in content for specialists in palliative care. The 24 sessions that remained comprised the final version of the learning path. This is now available on the e-ELCA website.

Conclusions The JRCPTB speciality training curriculum for palliative medicine was used to identify e-ELCA sessions that specialists in palliative care could use for their own education.

\section{P-257 A REVIEW OF THE (QELCA)@ (QUALITY AT END OF LIFE CARE FOR ALL) PROGRAMME ADAPTED FOR LEADERS AT ST CHRISTOPHER'S HOSPICE}

Anne Nash, Kim Briggs, Julie O'Neill. St Christopher's Hospice, London, UK

10.1136/bmjspcare-2018-hospiceabs.282

This programme, funded by the Burdett Trust, used an adapted version of the (QELCA) C) course. Its aim was to develop the leadership skills of inpatient managers. The group visited and observed external NHS leaders in both hospital 